\title{
SOME BOUNDS ON THE MODIFIED RANDIC INDEX
}

\author{
Mahsa Hemmasi ${ }^{1}$ and Ali Iranmanesh ${ }^{2 *}$ \\ ${ }^{1}$ Department of Mathematics, Science and Research Branch, Islamic Azad University, \\ P.O. Box 14515-1775, Tehran, I.R.Iran \\ E-mail: mahsa.hemmasi@gmail.com \\ ${ }^{2}$ Department of Mathematics, Tarbiat Modares University, \\ P.O. Box 14115-137, Tehran, I.R.Iran \\ E-mail: iranmanesh@modares.ac.ir
}

(Received June 6, 2014)

\begin{abstract}
In this paper, we present some new lower and upper bounds for the modified Randic index in terms of maximum, minimum degree, girth, algebraic connectivity, diameter and average distance. Also we obtained relations between this index with Harmonic and Atom-bond connectivity indices. Finally, as an application we computed this index for some classes of nano-structures and linear chains.
\end{abstract}

Keywords: Randic index, Girth, Harmonic index, $\mathrm{ABC}$ index, Algebraic connectivity, Average distance.

\section{INTRODUCTION}

In this paper $G=(V(G), E(G))$ is a simple connected graph, where $V(G)$ is the set vertex of $G$, and $\boldsymbol{E}(G)$ is the edge set of $G$. There are many different kinds of chemical indices that some of them are distance based like Wiener index, some of them are based on degree like Randic index. This fact is emphasized in the recent survey [12] which contains uniform approach to the degree- based indices.

The Randic index was proposed by Milan Randic in 1975. This topological index was named Branching index, later called Randic index, which defined as $R(G)=\sum_{u v E Z(G)} \frac{1}{\sqrt{d_{n u} d_{n}}}$, where $d_{u}$ denote the degree of vertex $u$. This index has been defined to measure the extent of branching of the carbon-atom skeleton of saturated hydrocarbons. Although Milan Randic showed that there is a good correlation between this index and physicochemical properties of alkanes such as boiling points, surface areas and energy levels [1, 3, 27]. There are many applications in organic chemistry, medicinal chemistry and pharmacology that this index became one of the most interesting topic in graph theory which 4 books are devoted $[10,18$ 19, 23]. In 2011, Z.Dvorak proposed a modified of Randic index, defined as $R^{r}(G)=\sum_{u v \in E[G]} \frac{1}{\max \left[d_{w} d_{v}\right]}$, that is more tractable from computational point of view. It is much easier to follow during graph modifications than Randic index see [5] for more details. In [2], the authors showed that for every graph with $n$ vertices, $R^{\prime}(G)$ is at least 1 no more than $\frac{\pi}{2}$ and these bounds attained by stars and regular graph. Although they determined graphs 
with minimal and maximal value of $R^{\prime}(G)$ among all trees and unicylic graphs, in [4], the authors showed that for all connected graph $G$ the inequality $R^{\prime}(G) \geq \operatorname{rad}(G)-\frac{1}{2}$ holds where $\operatorname{rad}(G)$ is the minimum eccentricity among all vertices of $G$, and the eccentricity of the vertex $v$ is the maximum distance from $v$ to any vertex. The maximum and minimim degree of a vertex in $G$ denoted by $\Delta(\mathrm{G})$ and $\delta(\mathrm{G})$, respectively.

The Laplacian matrix of $\mathrm{G}$ is defined as $L_{G}=D_{G}-A_{G}$, where $D_{G}$ is the diagonal matrix of its vertex degree and $A_{G}$ is the adjacency matrix. Among all eigenvalues of the Laplacian matrix of $\mathrm{G}$, one of the most popular is the second smallest, which was called the algebraic connectivity of a graph by fiedler [9 ] in 1973, and denoted by $a(C)$. In [22], the authors get relation between Randic index and algebraic connectivity. The girth of a graph G, denoted by $g(G)$ is the minimum length of its cycles. In [21] the authors computed upper bound of Randic index with girth $g$. Let $\mu(G)$ be the average distance of $\mathrm{G}$ that defined as $\mu(G)=\frac{w(G)}{\left(T_{2}\right)}$ such that $W(G)$ is the Wiener index defined as the sum of the lengths of the shortest path between all pairs of vertices and diameter of $G$ is the maximum distance over all pairs of vertices $u$ and $v$ of $G$ denoted by $D(G)$. In [30], the authors obtained relation between Randic index and diameter of a graph.

The edge cut of $G$ is a group of edges whose total removal renders the graph disconnected. The edge connectivity $\lambda(G)$ is the size of a smallest edge cut. In this paper, we obtain a new bounds for the modified Randic index in terms of girth, diameter and algebraic connectivity. In continue, we establish some relation between this index and harmonic index and ABC index. The harmonic index of graph $\mathrm{G}$ is defined as $H(G)=\Sigma_{w \in E(G)} \frac{2}{d_{u}+d_{y}}$. The atom-bond connectivity index of a nontrivial graph $\mathrm{G}$, denoted by $A B C(G)$, is defined as $A B C(G)=\sum_{w v E E(G)} \sqrt{\frac{d_{u}+d_{v}-1}{d_{u} d_{v}}}$. For more information about harmonic and ABC index we refer the reader to see $[7,11,26,29]$.

\section{MAIN RESULTS}

The aim of this section is to determine some new bounds for $R^{r}$ in terms of girth, diameter and algebraic connectivity minimum and maximum degree.

Theorem 2.1: Let $G$ be a connected triangle-free graph with $n \geq 2$ vertices and $m$ edges. Then we have:

$R^{\prime}(g) \geq \frac{m}{n}$

with equality if and only if $G$ is an (n+1)-vetrex star $S_{n+1}$.

Proof: Let $w v$ be an edge in $G$. Since $G$ is triangle-free, we have $d_{u} \leq n-d_{v}$. Therefore regarding the definition of $R^{\prime}(G)$, we have $R^{r}(G) \geq \frac{m}{n}$. Furthermore, If $G$ is the $(n+1)$ vertex $\operatorname{star} S_{n+1}$, then $R^{\prime}(G)=\frac{n-1}{n}=\frac{m}{n}$.

Conversely, we assume that $R^{\prime}(G)=\frac{m}{n}$ but $G$ is not isomorphic to $S_{n+1}$, then there must existan edge $w$ such that $d_{u} \leq(n \| 1) \quad d_{v}$, implying that $R^{S}(G)>\frac{m}{n}$, a contradiction. This completes the proof. 


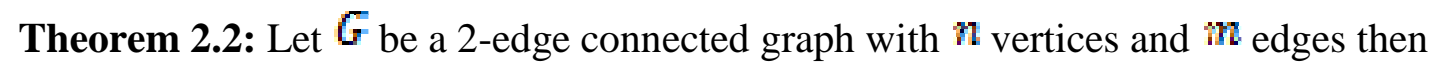
$\mathbb{R}^{\prime}(G) \leq \frac{m}{2}$

with equality if and only if $G$ is an $n$-vertex cycle $C_{\mathrm{n}}$.

Proof: Let $u$ be an edge in $G$. Since $G$ is 2 -edge connected, we have $d_{u} \geq 2$ and $d_{v} \geq 2$. So we have $R^{r}(G) \leq \frac{m}{2}$ with equality if and only if $d_{v}=2$ for any vertex $v$ in $G$, that is $G \cong C_{\mathrm{n}}$.

Note that a 2-connected graph is necessarily a 2-edge connected graph. By above theorem we have:

Corollary 2.3: Let $G$ be a 2-connected graph with $n$ vertices and $m$ edges then we have

$$
\mathrm{R}^{r}(\mathrm{G}) \leq \frac{\mathrm{m}}{2}
$$

With equality if and only if $G$ is an $n$-vertex cycle $C_{\mathrm{n}}$.

Let $S_{n}^{\text {th }}$ be a unicyclic graph obtained from star $K_{1_{i} n-1}$ by joining two pendant vertices of $K_{1, n-1}$ by a new edge. (Fig. 1)

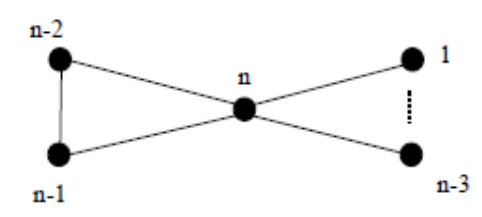

Fig. 1. The graph $S_{n}^{*}$

Theorem 2.4 : Let $\mathrm{G}$ be a connected graph on $n \geq 3$ vertices and girth $g$ with $\delta \geq 2$, then the following inequalities hold:

$$
R^{r}+g \geq \sqrt{2(n-3)}+\frac{2}{2} \cdot R^{r} \cdot g \geq 3 \sqrt{2(n-3)}-\frac{a}{2}
$$

The equality holds if $\mathrm{G} \cong S_{5}^{4}$.

Proof: In [5], the authors proved that

$$
R^{\prime}(G) \geq \sqrt{2(n-3)}-\frac{1}{2}
$$

It is easy to see that $F$ contains at least one cycle since minimum degree $\delta$ is at least 2 , so the girth of $G$ is at least 3 , this implies the inequality. Therefore the equalities hold if $\mathrm{G} \cong 5_{5}$, so the proof is now completed.

Lemma 2.5: Let $G$ be a graph on $n$ vertices with the algebraic connectivity $a_{\text {, we have: }}$

where $\lambda$ denotes the edge connectivity of $G$.

$$
a \geq 2 \lambda\left(1-\cos \frac{\pi}{n}\right) \text {, }
$$

Proof: see [9] for more details.

Theorem 2.6: Let $G$ be a graph on $n$ vertices and edge connectivity $\lambda \geq 2$ such that $d(G) \geq 3$, we get the following inequality:

and if $\lambda=1$ then we have:

$$
R^{r}, a \geq 4 \sqrt{2(n-3)}\left(1-\cos \frac{\pi}{n}\right)
$$

$$
R^{r} a \geq 2 \sqrt{2(n-3)}\left(1-\cos \frac{\pi}{n}\right)
$$


Proof: Due to the above Lemma, if the edge connectivity $\lambda \geq 2$, we have $a \geq 4\left(1-\cos \frac{\pi}{n}\right)$ and by using inequality (1) we can obtain:

$$
R^{r} \cdot a \geq 4 \sqrt{2(n-3)}\left(1-\cos \frac{\pi}{n}\right)
$$

To prove the second part, it is enough to apply lemma 2.5 .

Theorem 2.7: Fix a positive integer n. Among all trees on $n$ vertices and maximum degree $\Delta$, the maximum value of algebraic connectivity equals to $\Delta+2 \sqrt{\Delta-1}$.

Proof: See [28 ].

Theorem 2.8: Among all trees, the maximum value of modified Randic index equals to $\frac{n-a}{2}$.

Proof: See [2].

Theorem 2.9: Let $\mathrm{T}$ be a tree with $n$ vertices and algebraic connectivity $a$, the following ineqaulity holds:

$$
R^{\prime} \cdot a<\frac{n-3}{2}(\Delta+2 \sqrt{\Delta-1})
$$

Proof: Due to Theorem 2.7and 2.8, we have:

$$
a<\Delta+2 \sqrt{\Delta-1}, R^{\prime}(T) \leq \frac{\pi-1}{2}
$$

so we have:

$$
R^{\prime} \cdot a<\frac{n-3}{2}(\Delta+2 \sqrt{\Delta-1})
$$

Lemma 2.10 : Let $G$ be a connected graph with $n$ vertices and minimum degree $\delta \geq 2$. Then it follows that

Proof: see [6] for more details.

$$
D(c) \leq \frac{2 \pi}{g+1}-1
$$

Theorem 2.11: Let $\mathrm{G}$ be a connected gaph with $n$ verices and minimum degree $\delta \geq 2$. Then we get the following inequality:

$$
\frac{R^{*}}{D} \geq \frac{\sigma \sqrt{(\mathrm{n}-3)}-\frac{1}{2}}{3 n}
$$

Proof: By the Inequality (1) and the above Lemma we have:

$$
\frac{\mathbb{R}^{\prime}}{\mathrm{D}} \geq \frac{\sqrt{2(n-3)}-\frac{1}{2}}{\frac{3 n}{\delta+1}-1} \geq \frac{(\delta+1)}{3 n}\left(\sqrt{2(n-3)}-\frac{1}{2}\right) \geq \frac{\delta\left(\sqrt{n-3}-\frac{1}{2}\right)}{3 n}
$$

Lemma 2.12: If $G$ is a graph with $n$ vertices and minimum degree $\delta$, then we have:

Proof: See [20] for more details.

$$
\mu(G) \leq \frac{n}{d+1}+2
$$


Theorem 2.13: Let $G$ be a connected graph with $n$ vertices and minimum degree $\delta$ then it follows that

$$
\frac{R^{\prime}}{\mu} \geq \frac{\delta\left(\sqrt{(n-3)}-\frac{1}{2}\right.}{n+2(s+1)}
$$

Proof: By inequality (1) and the above Lemma we have

The proof is now complete.

$$
\frac{n^{\prime}}{\mu} \geq \frac{\sqrt{2(n-3)}-\frac{1}{2}}{\frac{n}{n+1}+2} \geq \frac{(\delta \| 1) \sqrt{2(n-3)}}{n+2(\delta+1)} \geq \frac{3\left(\sqrt{n-3}-\frac{1}{2}\right)}{n+2(\delta+1)}
$$

Now, we obtain relations between the modified Randic index, Harmonic and ABC indices.

Theorem 2.14: let $G$ be a nontrivial connected graph with $n$ vertices and $m$ edges then we have

$$
H(\sigma) \geq E^{\prime}(\sigma)
$$

Proof: Let $w$ be an edge in $G$. Since $\max \left(d_{u}, d_{v}\right)<d_{u}+d_{v}$, we have $\frac{1}{d_{u}+d_{v}}<\frac{1}{\max \left(d_{w} d_{v}\right)}$. So clearly we obtain:

$$
H(a) \geq R^{\prime}(a)
$$

Theorem 2.15: Let $G$ be a nontrivial connected graph on $n$ vertices and $m \mathrm{edges}$, then we have:

$$
H(\sigma) \leq \frac{1}{2}(A B C)^{2}+R^{\prime}(G)
$$

with equality if and only if $G \cong K_{2}$.

Proof: Let $f(x)=\frac{1}{x}$.It is obvious that $f(x)$ is a convex function. By Jensen's inequality, for each edge $m \in E(G)$, we have

$$
\frac{1}{\frac{1}{2}\left(d_{u}+d_{v}\right)} \leq \frac{1}{2}\left(\frac{1}{d_{u}}+\frac{1}{d_{w}}\right)
$$

with equality if and only if $d_{u}=d_{v}$. Since $0 \leq \frac{1}{d_{u}}+\frac{1}{d_{v}}-\frac{2}{d_{u} d_{v}}<1$, we have

thus we have

$$
\begin{aligned}
\frac{2}{d_{u}+d_{v}} & \leq \frac{1}{2}\left(\frac{1}{d_{u}}+\frac{1}{d_{v}}-\frac{2}{d_{u} d_{v}}\right)+\frac{1}{d_{u}+d_{v}} \\
& \leq \frac{1}{2}\left(\frac{1}{d_{u}}+\frac{1}{d_{v}}-\frac{2}{d_{u} d_{v}}\right)+\frac{1}{\max \left(d_{u} d_{v}\right)}
\end{aligned}
$$

$$
\frac{1}{d_{u}+d_{v}} \leq \frac{1}{2}\left(\frac{1}{u_{u}}+\frac{1}{u_{v}}-\frac{2}{u_{u} u_{v}}\right)+\frac{1}{\operatorname{mgx}\left(u_{w} u_{v}\right)} \leq \frac{d_{u}+d_{v}-2}{u_{u} u_{v}}+\frac{1}{\max \left(d_{u} A_{v}\right]}=\frac{1}{2}(A B C)^{2}+R^{r}(G) .
$$

The equality holds in the above inequality if and only if $d_{u}=d_{v}$.

\section{Computation of the modified Randic index of $\operatorname{TUZC}_{6}(p, q)$ nanotubes:}

A carbon nanotube is forming from a graphite sheet that is rolled up so that it has a zigzag edge. In this paper, we computed the modified Randic index for some families of polyhex nanotubes, armchair, Phenylenic Nanotorus, Polycyclic Aromatic Hydrocarbons and polyomino chain (Figs. 2-8). 
By considering the lattice of TUZC $_{6}[\mathrm{p}, \mathrm{q}]$, we denote the number of hexagon in the first row by $p$ and the number of rows by $q$. In each row, there are $2 p$ vertices and hence the number of vertices in this nanotube equals to 2pq. In [13] the authors obtained the hyper Wiener and Schultz indices of $\mathrm{TUZC}_{6}[\mathrm{p}, \mathrm{q}]$ nanotube, in [14] the authors computed GA index for this nanotube and in [8], the author computed some connectivity index and Zagreb index of nanotube. Now in this section, we compute the modified index of TUZC $_{6}[\mathrm{p}, \mathrm{q}]$.

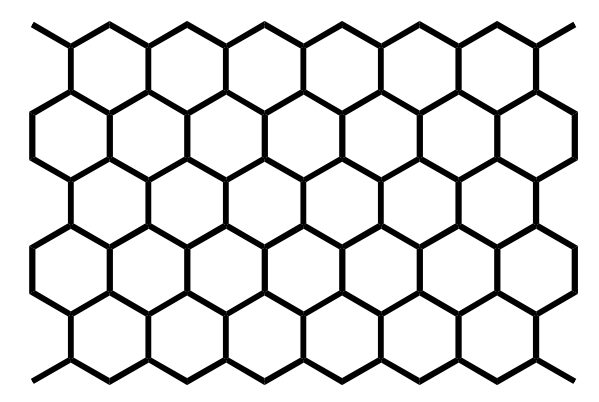

Fig. 2. The 2-Dimensional Lattice of TUZC $_{6}[7,6]$

Set $\int_{m n}=\left\{w v \| w v E E(G), d_{v}=w, d_{u}=n\right\}$, since in the graph of nanotube $\operatorname{TUZC}_{6}(\mathrm{p}, \mathrm{q})$, all

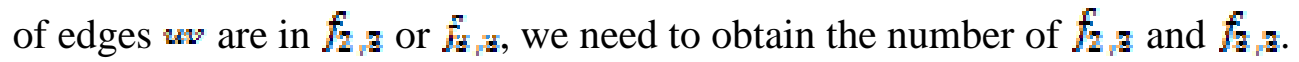

Lemma 3.1: The number of $f_{r}$ equals to $4 p$ and the number of $f_{z} a$ equals to $3 p q-5 p$.

Proof: Consider the $\mathrm{TUZC}_{6}[\mathrm{p}, \mathrm{q}]$ nanotube. At the first and last rows, there exist edges that every edge in these rows belong to $f_{z} a$, hence the number of $f_{z} z$ equals to $4 \mathrm{p}$. At the other rows there exist $p$ edges that belong to $f_{z}$ and the number of these edges are $q-1,2 p$ edges that every edges belong to $f_{z, a}$ and the number of these edges are $\mathrm{q}-2$, hence the number of $f_{n, 1}$ equals to $3 \mathrm{pq}-5 \mathrm{p}$.

Theorem 3.2 : The modified Randic index of $\mathrm{TUZC}_{6}[\mathrm{p}, \mathrm{q}]$ equals to $\frac{1}{2}(3 p q-p)$.

Proof: By using the modified Randic index formula and the number of edges with their degrees we have:

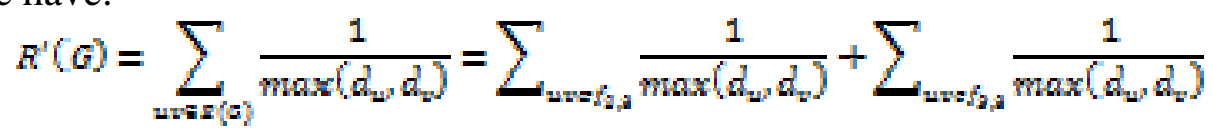

$$
\begin{aligned}
& =\frac{1}{2}(3 p q-5 p+4 p)=\frac{1}{2}(3 p q-p) .
\end{aligned}
$$

Now, we compute the modified Randic index of armchair TUAC $6[\mathrm{p}, \mathrm{q}]$ similar to previous section. The number of vertices in this armcheir equals to 2pq. The armchair's edges are in 3 types. The yellow edges belong to $f_{z}$, the red edges belong to $\hat{h}_{z}$ and the other edges belong to $f_{z, a}$. The number of $f_{2,2}, f_{2, a}$ and $f_{3, a}$ are equal to $\mathrm{p}, 2 \mathrm{p}$ and $3 \mathrm{pq}-4 \mathrm{p}$, respectively.

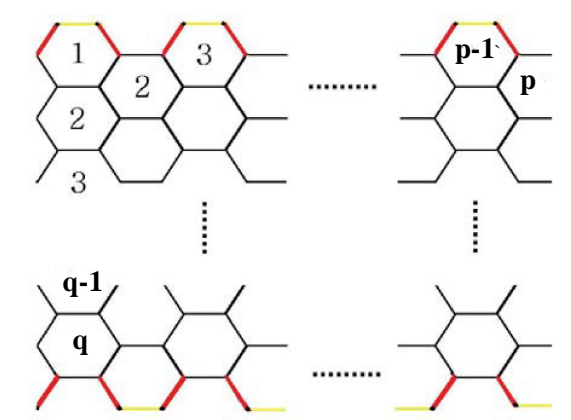

Fig. 3. The 2-Dimensional Lattice of $\mathrm{TUAC}_{6}[\mathrm{p}, \mathrm{q}]$ 
Theorem 3.3: The modified Randic index of $\mathrm{TUAC}_{6}[\mathrm{p}, \mathrm{q}]$ equals to $\mathrm{pq}-\frac{\mathrm{p}}{6}$.

Proof: Like the previous theorem we have:

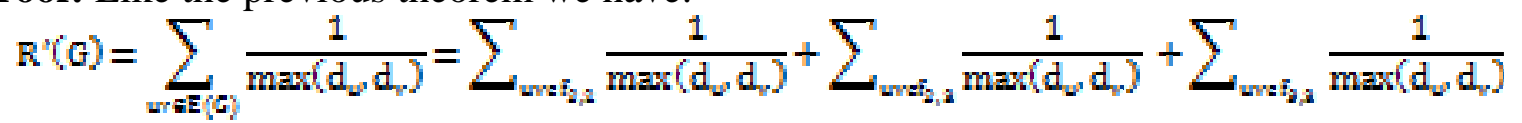

$$
\begin{aligned}
& =\frac{p}{2}+\frac{2 p}{a}+\frac{3 p q-4 p}{a}=p q-\frac{p}{6}
\end{aligned}
$$

The next goal of this section is a computing a closed formula of the modified Randic index of $\mathrm{TUC}_{4} \mathrm{C}_{8}[\mathrm{p}, \mathrm{q}]$ nanotube. In the structure of this nanotube there are pq horizontal regular squaroctagone lattice with $8 p q+2 p$ vertices and $12 p q+p$ edges (Fig. 4). For more results about this nanotube see $[15-17,24]$.



Fig. 4. 2-Dimensional Lattice of $\mathrm{TUC}_{4} \mathrm{C}_{8}[\mathrm{p}, \mathrm{q}]$ nanotube, with $\mathrm{p}=4$ and $\mathrm{q}=3$

Theorem 3.4: For $p, q \geq 1$, the modified Randic index of $\mathrm{TUC}_{4} \mathrm{C}_{8}[\mathrm{p}, \mathrm{q}]$ equals to $4 p q+\frac{2 p}{a}$.

Proof: Consider the Lattice of $\mathrm{TUC}_{4} \mathrm{C}_{8}[\mathrm{p}, \mathrm{q}]$ nanotube. In this nanotube we have three types of edges such that belong to $f_{2}, f_{2}$ and $f_{2}$ that are shown by red, blue and black colors such that $\| f_{2,2}|=2 p,| f_{2, a} \mid=4 p$ and $\left\|f_{a, z}\right\|=12 p q-5 p$. Thus we have

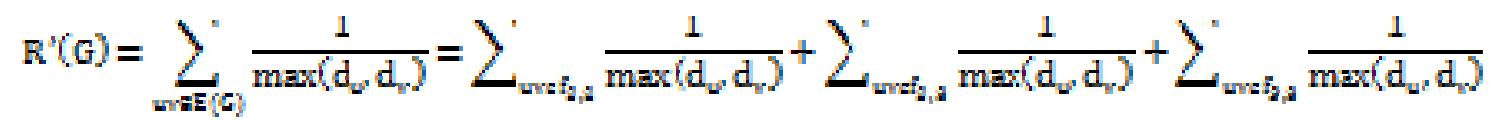

$$
\begin{aligned}
& =P+\frac{4 p}{3}+\frac{12 p q-5 p}{3}=4 p q+\frac{2 p}{3}
\end{aligned}
$$

In continue, we obtain $\mathrm{R}^{\prime}(\mathrm{G})$ of a physico chemical structure of Phenylenic Nanotorus. This nano structure is V-Phenylenic Nanotorus VPHY[p,q]. The structure of this nanotorus in terms of several $\mathrm{C}_{4} \mathrm{C}_{6} \mathrm{C}_{8}$ net that composed of four and six membered rings such that every square is adjacent to two hexagones (Fig. 5).

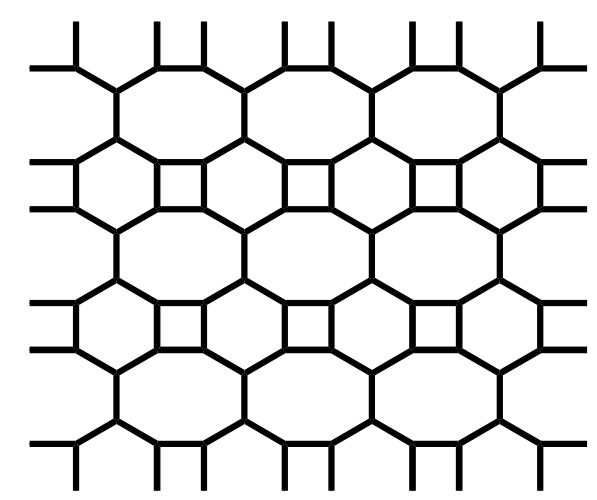

Fig. 5. The 2-Dimensional Lattice of VPHY[4,3] 
Theorem 3.5: For $p, q \geq 1$, the modified Randic index of V-Phenylenic Nanotori VPHY[p,q] equals to $\frac{\mathrm{pq}}{\mathrm{a}}$.

Proof: Due to the general Figure of V-Phenylenic Nanotori VPHY[p,q], this nanotori has 6pq vertices, 9pq edges and all edges belong to $\mathrm{f}_{3,3}$, this implies that

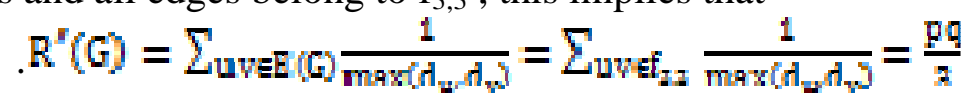

At the next goal, we calculate the modified Randic index of hydrocarbon structures Polycyclic Aromatic Hydrocarbons $\left(\mathrm{PAH}_{n}\right) . \mathrm{PAH}_{\mathrm{n}}$ are a complex group of chemicals containing two or more aromatic rings. $\mathrm{PAH}_{\mathrm{n}} \mathrm{s}$ are created when products like coal, oil, gas and garbage are burned but the burning process is not complete. The first member is Benzene $\left(\mathrm{PAH}_{1}\right)$ with six carbon and six hydrogen atoms and the second member is coronene $\left(\mathrm{PAH}_{2}\right)$ with 24 carbon and 12 hydrogen atoms (Fig. 6). By the Figure of the polycyclic aromatic hydrocarbon, it is easy to see that the general representation of has $P A H_{n} 6 \mathrm{n}^{2}$ carbon and $6 \mathrm{n}$ hydrogen atoms.
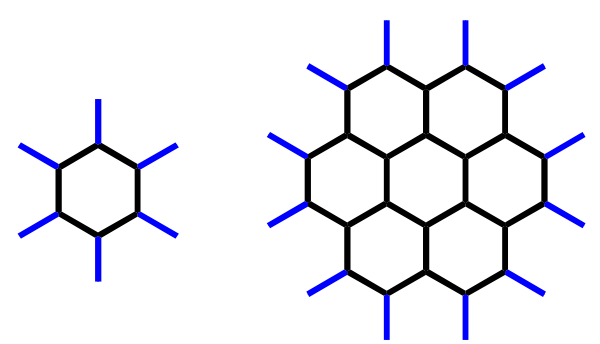

Fig. 6. The first and second member of polycyclic aromatic hydrocarbon $\mathrm{PAH}_{\mathrm{n}}$

Theorem 3.6: The modified Randic index of $P A H_{n}$ equals to $n+3 n^{2}$.

Proof: Let $P A H_{n}$ be the general representation of polycyclic aromatic hydrocarbon. The edge set of this graph can be dividing to two partitions, these partitions belong to $f_{1, z}, f_{2, a}$ and show that by blue and black color, respectively. All Carbon atoms have degree three and Hydrogen atoms have degree one, so we have $\left|f_{1, a}\right|-6 \mathrm{n}$ and $\left|f_{r_{i} a}\right|=9 \mathrm{n}^{2}-3 \mathrm{n}$, so we have:

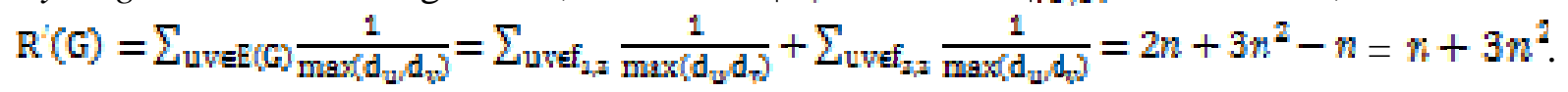

\section{The modified Randic index of polyomino chain:}

A polyomino system is a finite 2-connected plane graph such that each interior face is surrounded by a regular square of length one. A polyomino chain is a polyomino system, in which the joining of the centers of its adjacent regular forms a path $c_{1} c_{2} \ldots c_{n n}$ where $c_{i}$ is the center of the i-th square. Let $B_{n}$ be the set of polyomino chains with n squares, the subgraph of $B_{n}$ that induced by the vertices with degree 3 and n-2 squares, called a linear chain and denoted by $\boldsymbol{L}_{n}$ (Fig. 7). The subgraph of $\boldsymbol{B}_{\mathrm{n}}$ induced by the vertices with degree bigger than 2 be a path with n-1 edges, called a zig-zag chain and denoted by $Z_{n}$ (Fig. 8). In [31] the authors obtained Randic index of this graph.

A kink of a polyomino chain is any branched or angularly connected squares. A segment $\mathrm{S}$ of a polyomino chain is a maximal linear chain in the polyomino chains that include the kinks at its end. The number of squares in a segment denoted by $l(G)$.

A polyomino chains consist a sequence of segments $S_{1}, \ldots, S_{k}, k \geq 1$, with $\boldsymbol{t}\left(S_{i}\right)=l_{i}, i=1, \ldots, k$, where

$l_{1}+\ldots+l_{k}=n+k-1$ and $n$ denote the number of squares of polyomino chain. 


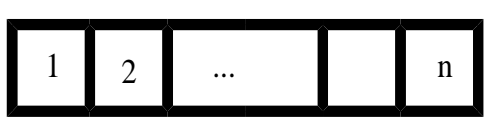

Fig. 7. The Linear chain

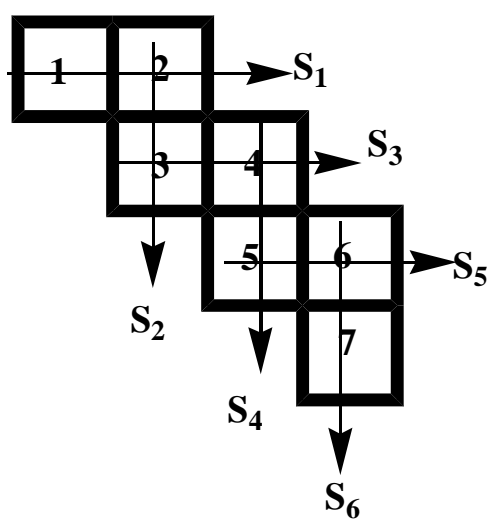

Fig. 8. The Zig-Zag chain

In the following, the aim is to calculate the Randic index of polyomino chains.

Theorem 4.1: Let $\mathbb{L}_{n}, Z_{n}$ be the polyomino chains then we have

$$
\mathrm{R}^{\prime} \rightarrow \mathrm{R}^{\prime}\left(L_{n}\right)=\left\{\begin{array}{cc}
2, & n=1 \\
\vdots, & n=2 \\
n+\frac{1}{z}, & n \geq 3
\end{array} \quad, \quad \mathrm{R}^{\prime} \rightarrow \mathrm{R}^{\prime}\left(Z_{n}\right)=\left\{\begin{array}{cc}
2, & n=1 \\
\frac{\pi}{z}, & n=2 \\
\frac{n-1 z}{2 z}, & n \geq 3
\end{array}\right.\right.
$$

Proof: For $n \leq 2$ it is trivial, we assume that $n \geq 3$. By the general Figure of $L_{n 1}$, the number of $f_{i \leq}$ equals to 2 , the number of $f_{2}$ equals to 4 and the number of $f_{z}$ e equals to $3 n-5$. By the definition of modified Randic index, we have



$$
\begin{aligned}
& =1+\frac{4}{3}+\frac{3 n-5}{3}=n+\frac{2}{3} \text {. }
\end{aligned}
$$

The edge set of $Z_{\mathrm{n}}$ with $n$ squares can be dividing to 5 partitions, these partitions belong to $f_{z, z}, f_{z, z}, f_{\pi, 4}, f_{A, 4}$ and $f_{4,4}$. Thus we have

$$
\mathbb{R}^{\prime}\left(z_{n}\right)=\Sigma_{\mathrm{WrE}}(c) \frac{1}{\max \left(d_{-} d_{n}\right)}=1+\frac{4}{3}+\frac{n-2}{2}+\frac{1}{2}+\frac{n-3}{4}=\frac{9 n-13}{12}
$$

Theorem 4.2: Let $B_{n}^{L}$ be a polyomino chain with $n \geq 3$ squares and $S_{1}, S_{2}$ segments, such that $l_{1}=2, l_{2}=n-1$. Then we have

$$
R^{\prime}\left(B_{n}^{1}\right)=\left\{\begin{array}{cc}
\frac{19}{2} & n=3 \\
n+\frac{1}{2} & n>3
\end{array}\right.
$$

Proof: For $n=3$ it is trivial, we assume that $n>3$. By considering the general Figure of $Z_{\text {n, we have }}$

$$
\left|f_{z,}\right|=2,\left|f_{z,}\right|=5,\left|f_{z, 4}\right|=1,\left|f_{z,+}\right|=3 \text { and }\left|f_{z, 2}\right|=a_{n}-10
$$


So we have

$$
R^{\prime}\left(B_{n}^{1}\right)=\sum_{u v E E(\omega)} \frac{1}{\max \left(d_{w} d_{v}\right)}=1+\frac{5}{3}+\frac{1}{4}+\frac{5}{4}+\frac{3 n-10}{3}=n+\frac{1}{3} .
$$

In following, we assume that $2 \leq l(i) \leq n-1$ such that $1 \leq i \leq k$.

Theorem 4.3: Let $B_{n}^{2}$ be a polyomino chain with $n \geq 4$ squares and $S_{1}, S_{2}, \ldots, S_{k}(k \geq 3)$ segments, such that $l_{1}=l_{k}=2, l_{2}, \ldots, l_{k-1} \geq 3$. Then

$$
R^{\prime}\left(B_{\pi}^{2}\right]=\left\{\begin{array}{cc}
\frac{i}{2} & n=4 \\
n-\frac{k}{z}+1 & n>4
\end{array}\right.
$$

Proof: For $n=4$ it is trivial. Therefore, we assume that $n>4$. By considering the structure of $B_{n, 2}^{2}$ we have $\left|f_{2,2}\right|=2,\left\|f_{2, a}\left|=2 k,\left\|\hat{h}_{2,4}|=2,| f_{2,4}\right\|=4 k-6\right.\right.$

and $\left|f_{z_{i}}\right|=3 n+1-2-2 k-2-(4 k-6)=3 n-6 k+3$.

Thus we have:

$$
R^{r}\left(B_{n}^{2}\right)=\sum_{w v E E[6]} \frac{1}{\left.\max ^{\prime} d_{w} d_{v}\right)}=1+\frac{2 k}{3}+\frac{2}{4}+\frac{4 k-6}{4}+\frac{3 n-8 k+3}{3}=n-\frac{k}{3}+1 .
$$

\section{CONCLUSIONS}

In this paper we achieved the lower and upper bounds for the modified Randic index in terms of girth, diameter and algebraic connectivity. Then we obtained a relation between this index with Harmonic and $\mathrm{ABC}$ indices. At the end of this paper we computed this index for some families of polyhex nanotubes $\operatorname{TUZC}_{6}[\mathrm{p}, \mathrm{q}], \mathrm{TUAC}_{6}[\mathrm{p}, \mathrm{q}], \mathrm{TUC}_{4} \mathrm{C}_{8}[\mathrm{p}, \mathrm{q}], \mathrm{VPHY}[\mathrm{p}, \mathrm{q}]$ nanotorus, Polycyclic Aromatic Hydrocarbons and polyomino chains for the first time.

\section{Acknowledgment}

The authors would like to thank the referee for his/her careful reading and useful suggestions which led us to improve the paper. Partial support by the Center of Excellence of Algebraic Hyper structures and its Applications of Tarbiat Modares University (CEAHA) is gratefully acknowledged by the second author (AI).

\section{References:}

[1] M. Aouchiche, P. HAnsen and M. ZHENG, Variable neighborhood search for extremal graphs. 19. further conjectures and results about the Randić index, MATCH Commun. Math. Comput. Chem. 58 (2007) 83-102.

[2] V. Andova, M. Knor, P. Potocnik and R. Skrekovski, On a variation of Randic index, IMFM Preprint Series, 49 (2011).

[3] G. CAPORossi and P. HAnsen, Variable neighbourhood search for extremal graphs 6. Analyzing bounds for the connectivity index, J. Chem. Inf. Comut. Sci. 43 (2003) 1-14.

[4] M. Cygan, M. PiLIPCZUK and R. SkReKovski, On the inequalioti between radius and Randic index for graphs, MATCH Commun. Math. Comput. Chem. 67 (2012) 451-466. 
[5] Z. DVORAK, B. Lidicky and R. SKREKOvski, Randic index and the diameter of a graph, Eur. J. Comb. 32 (2011) 434-442.

[6] P. Edros, J. Pach, R. Pollack and Z. TuZA, Radius, diameter, and minimum degree, J.Combin. Theory Ser. B 47 (1989) 73-79.

[7] E. Estrada, L. Torres, L. Rodríguez and I. Gutman, An atom-bond connectivity index. Modelling the enthalpy of formation of alkanes. Indian Journal of Chemistry 37A (1998) 849-855.

[8] M. Farahani, Some Connectivity Indices and Zagreb Index of Polyhex Nanotubes, Acta Chim. Slov. 59 (2012) 779-783.

[9] M. FIEDLER, Algebra connectivity of graphs, Czech. Math. J. 23 (1973) 298-305.

[10] I. Gutman and B. FuRTUla (Eds.), Recent Results in the Theory of Randic Index, Univ. Kragujevac, Kragujevac (2008).

[11] I. Gutman, J. Tošović, S. RADEnković and S. Marković, On atom-bond connectivity index and its chemical applicability, Indian J. Chem. 51A (2012) 690-694.

[12] I. Gutman, Degree-based topological indices, Croat. Chem. Acta 86 (2013) 351-361.

[13] A. Iranmanesh and Y. AlizadeH, Computing Hyper Wiener and Schultz indices of TUZC $_{6}[\mathrm{p}, \mathrm{q}]$ nanotube by GAP Program. Digest Journal of Nanomaterials and Biostructures 4 (4) (2009) 607-611.

[14] A. Iranmanesh and M. ZeraAtKar, Computing GA index for some nanotubes, Optoelectronics and Advanced Materials. Rapid Communication 4 (11) (2010) 18521855.

[15] A. IRANMAnesh and A.S. KAFRAni, Computation of the first edge-Wiener index of $\mathrm{TUC}_{4} \mathrm{C}_{8}(\mathrm{~S})$ nanotube, MATCH Commun. Math. Comput. Chem. 62 (2009) 311-352.

[16] A. IRANMANESH and A. R. ASHRAFI, Balaban index of an armchair polyhex, $\mathrm{TUC}_{4} \mathrm{C}_{8}(\mathrm{R})$ and $\mathrm{TUC}_{4} \mathrm{C}_{8}(\mathrm{~S})$ nanotorus, J. Comput. Theor. Nanosci. 4 (2007) 514-517.

[17] A. Karimi, A. Iranmanesh and A. Tehranian, Computing a new edge-Wiener index of TUC4C8(s) nanotubes and $\mathrm{TUC}_{4} \mathrm{C}_{8}(\mathrm{R})$ nanotorus, Optoelectron. Adv. Mater. Rapid Commun. 4 (2010) 1856-1859.

[18] L.B. KIER and L.H. HALL, Molecular Connectivity in Chemistry and Drug Research, Academic Press, New York (1976).

[19] L.B. KIER and L.H. HALL, Molecular Connectivity in Structure-Activity Analysis, Research Studies Press-Wiley, Chichester (UK) (1986).

[20] M. KouIDER and P. WinKLER, Mean distance and minimum degree, J. Graph Theory 25 (1997) 95-99.

[21] M. LiAnG and B. LiU, On the Randic index and girth of graphs, Discrete Mathematics 161 (2013) 212-216.

[22] X. LI, Y. SHI and L. WANG, On a relation between Randic index and algebraic connectivity, MATCH Commun. Math. Comput. Chem. 68 (2012) 843-849.

[23] X. Li and I. Gutman, Mathematical Aspects of Randic-Type Molecular Structure Descriptors, Mathematical Chemistry Monographs No.1, Kragujevac (2006).

[24] A. Mahmiani, O. Khormali, A. Iranmanesh and M. YousefidaZ, The computation of total Szeged index of TUC4C8(R) nanotube, Optoelectron. Adv. Mater. Rapid Commun. 5 (2010) 2185-2189. 
[25] K.L. PATRA and A.K. LAL, The effect on the algebraic connectivity of a tree by grafting or collapsing of edges, Lin. Algebra Appl. 428 (2008) 855-864.

[26] M. RAnDIC, The connectivity index 25 years after, J. Mol. Graphics Modell. 20 (2001) 19-35.

[27] M. RANDIC, On characterization of molecular branching, J.Amer. Chem. Soc. 97 (1975) 6609-6615.

[28] D. Stevanović, Bounding the largest eigenvalue of trees in terms of the largest vertex degree, Linear Algebra Appl. 360 (2003) 35-42.

[29] X. XU, Relationships between harmonic index and other topological indices, Applied Mathematical Sciences 6 (2012) 2013-2018.

[30] Y. YANG and L. LU, The Randic index and the diameter of graphs, Discrete Mathematics, 311 (2011) 1333-1343.

[31] J. Yang, F. XIA and S. Chen, On the Randic Index of Polyomino Chains, Applied Mathematical Sciences 5 (2011) 255-260. 